\title{
The pleasures of play: pharmacological insights into social reward mechanisms
}

\author{
Viviana Trezza $^{1,3}$, Petra J.J. Baarendse ${ }^{1}$ and Louk J.M.J. Vanderschuren ${ }^{1,2}$ \\ ${ }^{1}$ Department of Neuroscience and Pharmacology, Rudolf Magnus Institute of Neuroscience, University Medical Center Utrecht, \\ Utrecht, The Netherlands \\ ${ }^{2}$ Department of Animals in Science and Society, Division of Behavioural Neuroscience, Faculty of Veterinary Medicine, Utrecht \\ University, Utrecht, The Netherlands \\ ${ }^{3}$ Department of Biology, University 'Roma Tre', Rome, Italy
}

\begin{abstract}
Like human children, most young mammals devote a significant amount of time and energy playing together, and social play is fun. Although social play is very pleasurable, it is more than just a frivolous activity: it is crucial for the development of behavioral flexibility, the acquisition of social and cognitive competence, and the maintenance of group cohesion. Social play is a natural reinforcer, and the neurotransmitter systems intimately implicated in the motivational, pleasurable and cognitive aspects of natural and drug rewards, such as opioids, endocannabinoids, dopamine and norepinephrine, play an important modulatory role in the performance of social play. In this review, we address the notion that social play is rewarding, and discuss recent developments in the neuropharmacology of this behavior. This provides a framework to understand how the brain processes social emotions, to make young individuals enjoy social play.
\end{abstract}

\section{Introduction}

Social behaviors, such as affiliative, sexual, parental and aggressive territorial behaviors, are an integral part of the mammalian behavioral repertoire essential for survival of the individual, group or species. Social play behavior, also referred to as 'rough and tumble play', is the earliest form of mammalian social behavior that is not directed at the mother, but at peers [1-3]. Similar to human children, most young mammals spend a substantial part of maturation engaging in play with peers, such as running, chasing, climbing and play fighting. The ability to engage in social play is one of the principal indicators of healthy development, both in animals and humans. Conversely, social play deficits are a core symptom of neuropsychiatric disorders in childhood and adolescence, such as autism, early-onset schizophrenia, and attention-deficit/hyperactivity disorder (ADHD) [4-6].

'Playing' and 'having fun' are almost synonymous. Indeed, perhaps the most prominent characteristic of social play is its high reward value [1-3]. However, although there seems to be no obvious direct function of play other than having fun, social play does have clear

Corresponding authors: Trezza, V. (vtrezza@uniroma3.it); Vanderschuren, L.J.M.J. (l.j.m.j.vanderschuren@umcutrecht.nl). benefits. By varying, repeating, and/or recombining subsequences of behavior outside their primary context, play serves to develop physical, cognitive and social capacities, and especially to acquire the ability to flexibly use these capacities under changeable circumstances [3,7]. More specifically, play experience in a social context is crucial for the development of normal socioaffective responses and the acquisition of social skills [8,9]. Social play might also serve to facilitate the development of other, non-social, cognitive capacities. Besides these developmental functions, social play has stress-reducing effects, and serves to maintain group cohesion [3]. These latter properties, as well as maintenance of the skills previously acquired through play, are probably the functions of social play behavior in adult animals [3].

Social play is characterized by its vigorous appearance, exaggerated forms of behavior, and high level of affiliation. The most detailed and extensive experimental research investigating mammalian social play has been performed in the laboratory rat (Rattus norvegicus) [1-3,10]. As in almost every mammalian species studied, rats engage in social play according to an inverted U-shaped curve in ontogeny, peaking during the juvenile period and then falling off around puberty [1-3]. Patterns of adult affiliative, sexual and aggressive behavior can be recognized in social play, although young and adult social behavior differ in intensity, form and contextual settings [2,3]. In rats, a bout of social play behavior starts with one rat soliciting (pouncing) another animal, by attempting to nose or rub the nape of its neck (Figure 1a). The animal that is pounced upon can respond in different ways: if the animal fully rotates to its dorsal surface, 'pinning' is the result (Figure 1b). Pinning is regarded as the most characteristic posture in social play in rats. As rats mature into adulthood, the structure of social play changes. Most prominently, the response to pouncing with full rotation to the dorsal surface (resulting in pinning) occurs less, and is replaced by evasions and partial rotations [3] (see Table 1 for a detailed description of the different components of social play behavior in rats).

Just like other naturally rewarded behaviors that are important for development and survival, such as feeding, drinking, and sexual behavior, social play is a reinforcer 


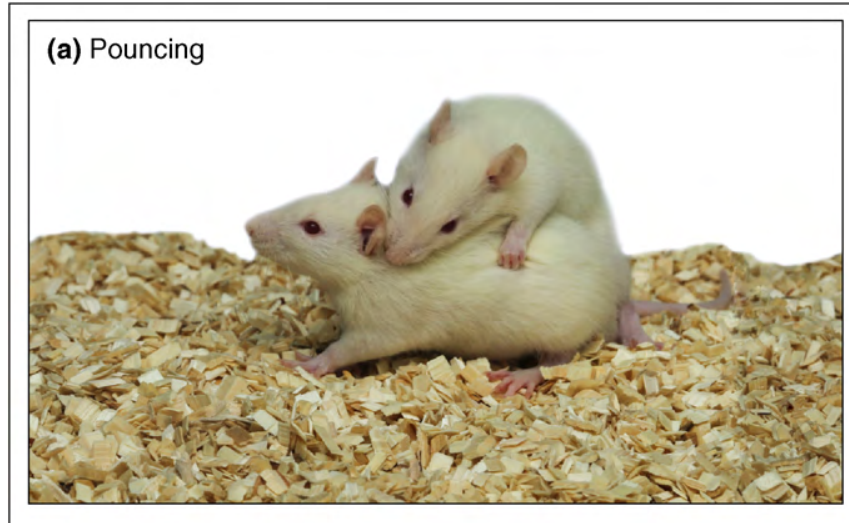

(b) Pinning

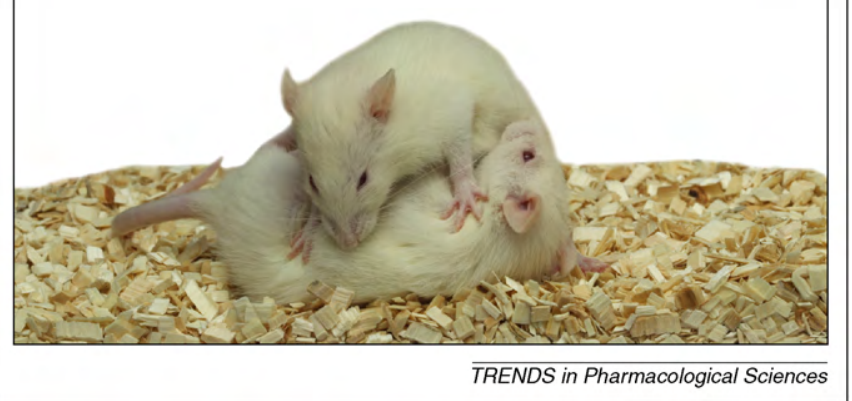

Figure 1. The most characteristic postures of social play in young rats. (a) Pouncing; (b) pinning. For a detailed description of the different components of social play behavior in rats, see Table 1.

both in rats and non-human primates [11-22]. Research into the neurobiological underpinnings of social play behavior has provided a substantial body of evidence that it is modulated by the neural systems that also mediate the positive subjective and motivational properties of food, sex and drugs of abuse. In this review, we provide an overview of recent advances in the neuropharmacology of social play behavior. We will restrict ourselves to studies in rats, because the vast majority of pharmacological studies on social play behavior has been performed in this species (see $[23,24]$ for examples of pharmacological studies in hamsters and monkeys). We focus on opioids, cannabinoids, dopamine and norepinephrine, the neurotransmitter systems that modulate the rewarding, motivational and cognitive aspects of this behavior (Table 2). Comprehensive overviews of pharmacological studies on social play, addressing the role of other neurotransmitters, such as gamma aminobutyric acid (GABA), acetylcholine and adenosine, can be found in previous reviews $[2,10]$.

\section{Rewarding properties of social play}

The earliest demonstrations of social reinforcement came from studies in primates, which showed that a chimpanzee learned a discrimination task that was rewarded by the opportunity to groom the experimenter [21]. The reinforcing properties of social play were subsequently shown by Mason et al. [22]. In this study, chimpanzees had the choice of pressing one of two levers. Pressing one lever produced food, and pressing the other lever produced social interaction. The experimenters manipulated the incentive value of the food reward by testing the animals when hungry or sated, or by offering foods of different palatability. The social interaction on offer was either petting or play. Food was chosen more often when the animals were hungry and when the most preferred food was available. Of the social behaviors, play was more reinforcing than petting. Intriguingly, even when the animals were tested hungry, they chose play on almost half of the occasions. When tested sated and offered foods of different palatability, the animals still chose play on half the occasions, even when the food reinforcer was very palatable. This pioneering study showed that social play is a powerful reinforcer in chimpanzees, comparable on a reward basis with tasty food. Rewarding properties of social play behavior in rats have since been demonstrated using both T-maze discrimination tasks $[11,16,17]$ and place conditioning [12-15,1820]. Rats will readily learn a T-maze discrimination task when rewarded with a social interaction, most prominently social play. Indeed, interactions with a partner that has been rendered non-playful (but not non-social) by physical confinement or drug treatment are less rewarding than playful social interactions [11]. It has repeatedly been shown that social play behavior can induce conditioned place preference [12-15,18-20]. Place conditioning studies have provided further evidence that it is indeed social play, rather than social interaction in general, that is rewarding, because interactions with non-playful animals did not induce conditioned place preference $[12,15]$. In addition, it has been shown that the positive subjective properties of cocaine or nicotine and social play can act synergistically, even if social play is reduced by these drugs [13,14]. Another line of empirical evidence to support the notion that social play is rewarding comes from studies showing that during social play, rats emit high frequency $(\sim 50 \mathrm{kHz})$ vocalizations [25] that are also emitted during other pleasurable events, such as sexual behavior and drug-induced conditioned place preference. Together, these data indicate that social play is rewarding, in the sense that it can be used as an incentive for maze learning, lever pressing and

Table 1. Ethogram of the social repertoire of young rats.

\begin{tabular}{|c|c|}
\hline Behavior & Description \\
\hline Pouncing & Nuzzling the nape of the conspecific's neck with the tip of the snout, followed by a rubbing movement (Figure 1a) \\
\hline Evasion & $\begin{array}{l}\text { Upon solicitation, the recipient animal avoids contact with the nape by leaping, running, or turning away } \\
\text { from the partner }\end{array}$ \\
\hline Partial rotation & $\begin{array}{l}\text { Upon contact of the nape, the recipient animal begins to rotate along its longitudinal axis, but then stops and keeps } \\
\text { one or both hind feet firmly planted on the ground }\end{array}$ \\
\hline Pinning & $\begin{array}{l}\text { Upon contact of the nape, the recipient animal fully rotates around the longitudinal axis of its body, ending in a supine } \\
\text { position with the other subject standing over it (Figure } 1 \mathrm{~b} \text { ) }\end{array}$ \\
\hline Boxing/wrestling & $\begin{array}{l}\text { Rearing in an upright position towards the other subject, combined with both rats rapidly pushing, pawing, } \\
\text { and grabbing at each other, or one rat wrapping around the other subject. }\end{array}$ \\
\hline Following/Chasing & Moving or running forward in the direction of or pursuing the other subject, who moves away \\
\hline Social exploration & Sniffing, licking or grooming any part of the body of the test partner, including the anogenital area \\
\hline
\end{tabular}


Table 2. Effects of opioid, cannabinoid, dopaminergic and noradrenergic drugs on social play behavior in adolescent rats.

\begin{tabular}{|c|c|c|c|}
\hline \multirow{2}{*}{$\frac{\text { Drug }}{\text { Opioids }}$} & \multirow[t]{2}{*}{ Pharmacodynamics } & Effect Antagonism & \multirow[t]{2}{*}{ Refs } \\
\hline & & & \\
\hline Morphine & $\mu$-Opioid receptor agonist & Naloxone, SR1417161A & {$[26,28,29,30,32,33,52]$} \\
\hline Methadone & $\mu$-Opioid receptor agonist & - & [2] \\
\hline Fentanyl & $\mu$-Opioid receptor agonist & $\uparrow$ & [35] \\
\hline$\beta$-endorphin & Endogenous $\mu$-opioid receptor agonist & $\uparrow$ & {$[52]$} \\
\hline Naloxone & Opioid receptor antagonist & $\downarrow$ & {$[31,28,34]$} \\
\hline Naltrexone & Opioid receptor antagonist & $\downarrow$ & [52] \\
\hline$\beta$-funaltrexamine & $\mu$-Opioid receptor antagonist & $\downarrow$ & [35] \\
\hline U50,488H & к-Opioid receptor agonist & $\downarrow$ & [35] \\
\hline Nor-binaltorphimine & к-Opioid receptor antagonist & $=$ & [35] \\
\hline BUBUC & $\delta$-Opioid receptor agonist & $=$ & [35] \\
\hline Naltrindole & $\delta$-Opioid receptor antagonist & $=$ & [35] \\
\hline \multicolumn{4}{|l|}{ Cannabinoids } \\
\hline WIN55,212-2 & CB1 cannabinoid receptor agonist & SR141716A & {$[32,33]$} \\
\hline Methanandamide & Endogenous cannabinoid receptor agonist & SR141716A & [34] \\
\hline SR141716A & CB1 cannabinoid receptor antagonist & $\downarrow$ & [34] \\
\hline URB597 & Anandamide hydrolysis inhibitor & Naloxone, SR1417161A, & I $[32,33]$ \\
\hline VDM11 & Endocannabinoid reuptake inhibitor & Naloxone, SR1417161A, & [ [34] \\
\hline AM404 & Endocannabinoid reuptake inhibitor & 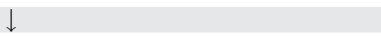 & [34] \\
\hline \multicolumn{4}{|l|}{ Dopamine } \\
\hline Apomorphine & Dopamine receptor agonist & $\uparrow$ & {$[51,54]$} \\
\hline Apomorphine & Dopamine receptor agonist & $\downarrow$ & {$[52]$} \\
\hline Quinpirole & Dopamine D2 receptor agonist & Eticlopride & [53] \\
\hline$\alpha$-Flupenthixol & Dopamine receptor antagonist & 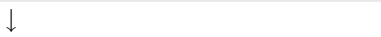 & [34] \\
\hline Chlorpromazine & Dopamine receptor antagonist & $\downarrow$ & {$[11,51]$} \\
\hline Haloperidol & Dopamine receptor antagonist & $\downarrow$ & {$[51,52]$} \\
\hline Eticlopride & Dopmaine D2 receptor antagonist & $\downarrow$ & {$[53]$} \\
\hline GBR-12909 & Dopmaine reuptake inhibitor & $=$ & {$[54]$} \\
\hline \multicolumn{4}{|c|}{ Dopamine/norepinephrine } \\
\hline Amphetamine & Dopamine/norepinephrine releaser and reuptake inhibitor & $\downarrow$ & {$[51,55,56]$} \\
\hline Methylphenidate & Dopmaine/norepinephrine reuptake inhibitor & $\mathrm{RX} 821882$ & {$[51,54,55,56]$} \\
\hline Cocaine & Dopamine/noradernaline/serotonin reuptake inhibitor & $\downarrow$ & [13] \\
\hline \multicolumn{4}{|l|}{ Norepinephrine } \\
\hline Atomoxetine & Norepinephrine reuptake inhibitor & $\mathrm{R} \times 821882$ & [54] \\
\hline Ephedrine & $\alpha-, \beta$-Adrenoceptor agonist & $\downarrow$ & {$[51]$} \\
\hline Phenoxybenzamine & $\alpha$-Adrenoceptor antagonist & $\downarrow$ & {$[51]$} \\
\hline Propranolol & $\beta$-Adrenoceptor antagonist & $\downarrow$ & {$[51]$} \\
\hline St-587 & $\alpha 1$-Adrenoceptor agonist & $=$ & {$[60]$} \\
\hline Prazosin & $\alpha 1$-Adrenoceptor antagonist & St-587 & {$[60]$} \\
\hline Clonidine & $\alpha 2$-Adrenoceptor agonist & Yohimbine & {$[51,59]$} \\
\hline Yohimbine & $\alpha 2$-Adrenoceptor antagonist & $=$ & [59] \\
\hline $\mathrm{R} \times 821002$ & $\alpha 2$-Adrenoceptor antagonist & $\uparrow$ & {$[60]$} \\
\hline
\end{tabular}

place conditioning, paradigms that have been widely used to study the rewarding properties of food, sex and drugs.

\section{Neuropharmacology of social play Opioids}

The idea that the endogenous opioid system is involved in the modulation of social play behavior emerged from the 'opioid theory of social behavior' postulated in the early $1980 \mathrm{~s}$ [26]. Based on putative analogies between social bond formation and opiate addiction, the core of this theory was that the pleasurable aspects of social behaviors are mediated by activation of the endogenous opioid system. Experimental support for this theory came from studies showing that endogenous opioids mediate infant attachment behavior and the rewarding properties of motherrelated stimuli, sexual behavior, social behavior and social play [27].

Opioids modulate social play through $\mu$ - and к-opioid receptors, with opposite behavioral outcomes. Thus, low doses of morphine, preferentially acting on $\mu$-opioid receptors, enhanced social play behavior in adolescent rats, whereas the nonselective opioid receptor antagonist naloxone suppressed it [28-34]. Interestingly, the playenhancing effect of morphine has also been demonstrated in primates [24]. More selective $\mu$-opioid receptor agonists and antagonists also increased and decreased social play, respectively $[2,35]$. Conversely, activation of $\kappa$-opioid receptors decreased social play, whereas $\delta$-opioid receptor stimulation had no effect [35]. It is noteworthy that opioids selectively increased social play without altering its sequential structure, and without changing locomotor activity or social behaviors unrelated to play [29].

The endogenous opioid system is a key regulator of reward processes, and as outlined above, social play is a very rewarding activity. Both natural and drug rewards can be dissociated in terms of their motivational (wanting), hedonic (liking) and cognitive (learning) properties [36,37]. The experimental setups that are commonly used to study social play behavior in adolescent rats do not allow for an accurate distinction between these components of social play. However, the available data suggest that opioids increase the hedonic properties of social play, rather than its motivational aspects. First, neither morphine nor naloxone affected play motivation in a T-maze task 
rewarded by social play. However, morphine increased and naloxone decreased the amount of social play performed in the goal box of the T-maze [17]. Second, in line with the idea that dopaminergic neurotransmission mediates the incentive motivational properties of rewards [38-40], whereas the endogenous opioid system also mediates their hedonic, pleasurable properties [36,38-40], the increase in social play induced by morphine was blocked by pretreatment with naloxone, but unaffected by the dopamine receptor antagonist $\alpha$-flupenthixol [32]. Third, opioid activity within the nucleus accumbens is thought to modulate the hedonic properties of food, whereas in the ventral tegmental area, opioids might enhance its motivational properties by indirectly stimulating mesolimbic dopaminergic neurotransmission [36,38-40]. In keeping with the notion that opioids modulate the hedonic, rather than the motivational aspects of social play, it has been shown that during social play, endogenous opioid activity was increased in the nucleus accumbens but not in the ventral tegmental area [41].

Neither morphine nor naloxone affected the rate of learning in the social play-rewarded T-maze task [17], but opioid neurotransmission could possibly modulate some of the cognitive aspects of social play. Indeed, treatment with either a low dose of morphine or with the $\mathrm{k-}$ opioid receptor antagonist nor-binaltorphimine attenuated the transient reduction in social play induced by a novel environment, suggesting that opioids induce a shift in selective attention as a result of altered integration of sensory information $[2,30,35]$.

In conclusion, there is a great deal of evidence to support an important role for the endogenous opioid system in the hedonic, and possibly cognitive, aspects of social play behavior.

\section{Cannabinoids}

Cannabinoid neurotransmission can have both positive and negative effects on social play behavior in adolescent rats, depending on how the endocannabinoid system is stimulated. Activation of CB1 cannabinoid receptors with direct cannabinoid agonists reduced social play behavior in rats [32-34], which is consistent with demonstrations that direct cannabinoid receptor agonists reduce social interaction in rats $[42,43]$. By contrast, indirect cannabinoid agonists, which enhance endocannabinoid activity by interfering with endocannabinoid degradation or reuptake [44], increased social play [32-34].

The explanation for these seemingly paradoxical effects of direct and indirect cannabinoid agonists on social play probably lies in the peculiar properties of endocannabinoid neurotransmission. Endocannabinoids are synthesized and released on demand after neuronal depolarization [44]. Thus, inhibiting their deactivation prolongs endocannabinoid signaling in active synapses only, preserving the spatiotemporal specificity of endocannabinoid activity. The effects of indirect cannabinoid agonists therefore suggest that, during social play, endocannabinoids are released in brain areas mediating this behavior. This endocannabinoid activity facilitates social play, so that drugs that prevent endocannabinoid degradation enhance play behavior by prolonging and magnifying endocannabinoid tone.
Conversely, direct cannabinoid agonists might also stimulate CB1 cannabinoid receptors in brain areas in which increased cannabinoid activity inhibits social play, for example, through the well-known disruptive effects of cannabinoid agonists on working memory and behavioral flexibility [45], causing a cognitive state that is incompatible with the adequate execution of complex social acts. Indeed, consistent with the notion that cannabinoid agonists disrupt cognition in the social domain, direct activation of CB1 cannabinoid receptors has been shown to impair social memory [46].

Regarding the mechanisms underlying cannabinoid modulation of social play, an interesting interaction between opioid and cannabinoid neurotransmission has emerged. The effects of indirect cannabinoid agonists on social play were not only blocked by the CB1 cannabinoid receptor antagonist SR141716A, but also by naloxone. Conversely, the play-enhancing effects of morphine were attenuated by both naloxone and SR141716A [32]. Thus, opioid-cannabinoid interactions, which play an important part in both drug and food reward [47,48], also mediate social reward. The effects of endocannabinoids and opioids on social play, however, are not identical. At the behavioral level, endocannabinoids enhanced responsiveness to play solicitation, but only when reciprocated by an equally socially motivated partner, suggesting that the increase in social play caused by augmented endocannabinoid activity was influenced by the level of social activity of the test partner. However, this was not the case for morphine, which enhanced social play independent of the playful responsiveness of the test partner [33]. At the pharmacological level, indirect cannabinoid agonists enhanced social play through a dopamine-dependent mechanism, whereas the effect of morphine on social play was dopamine-independent [32]. Interestingly, although cannabinoid neurotransmission was involved in the playenhancing effects of both nicotine and ethanol [49], ethanol-induced stimulation of social interactions had opioiddependent and opioid-independent components [49,50]. This further supports the notion that cannabinoid and opioid neurotransmission interact in the modulation of social play, but through distinct behavioral and neural mechanisms.

\section{Dopamine}

Because social play is a natural reward, it would be expected that drugs that increase dopaminergic neurotransmission enhance social play. However, the role of dopaminergic neurotransmission in the modulation of social play is less straightforward than this. Dopamine receptor antagonists reduce social play $[34,51,52]$, whereas both increases and decreases in social play have been reported after treatment with dopamine receptor agonists, with the reported increases being very modest $[51,53,54]$. The psychostimulants amphetamine, methylphenidate and cocaine, which indirectly increase dopaminergic neurotransmission by inhibiting dopamine reuptake, suppressed social play [13,51,54-56]. However, these drugs also inhibit the re-uptake of norepinephrine and serotonin, so that neurotransmitters other than dopamine might underlie their effects on social play. Indeed, as outlined 
below, the play-suppressant effects of methylphenidate are dependent on noradrenergic neurotransmission. Furthermore, selective inhibitors of norepinephrine and serotonin uptake reduced social play [54,57], whereas selective blockade of dopamine reuptake did not affect social play [54]. Because increases in forebrain dopamine turnover have been found during social play [58], it is possible that dopamine signaling is optimum when social play is performed, so that further stimulation of dopaminergic neurotransmission does not necessarily result in increased social play. These findings are reminiscent of studies on eating, which generally report that in a free feeding situation, changes in dopaminergic neurotransmission do not alter food intake. Dopaminergic modulation of feeding only emerges when the motivational properties of feeding are explicitly addressed [36,38-40]. The modulatory role of dopaminergic neurotransmission in social play is further supported by data showing that the increase in social play induced by endocannabinoids, nicotine and ethanol depends on dopaminergic neurotransmission $[32,49]$.

\section{Norepinephrine}

Social play behavior is affected by several drugs that target noradrenergic neurotransmission. Early studies have shown that the nonselective adrenoceptor agonist ephedrine, the $\alpha$-adrenoceptor antagonist phenoxybenzamine, the $\beta$-adrenoceptor antagonist propranolol, the $\alpha-1$ adrenoceptor antagonist prazosin, and the $\alpha-2$ adrenoceptor agonist clonidine strongly decreased social play $[51,59,60]$. These findings should, however, be interpreted with caution, for two reasons. First, relatively high doses of the drugs were used, which makes it difficult to disentangle specific drug effects on play from more general changes in locomotion or arousal. Second, neurochemical specificity has to date been demonstrated only for clonidine and prazosin, the effects of which were partially antagonized by the $\alpha-2$ adrenoceptor antagonist yohimbine and the $\alpha-1$ adrenoceptor agonist St-587, respectively $[59,60]$.

More information about the role of noradrenergic neurotransmission in social play has come from studies with indirect noradrenergic agonists. Particularly interesting are the effects of the psychostimulant methylphenidate, the first-choice medication for ADHD. Similar to amphetamine $[51,55,56]$, methylphenidate suppressed social play behavior at doses that did not affect locomotor activity or social behavior in general [54-56]. Methylphenidate reduced both the initiation to play and the responsiveness to play initiation, without inducing tolerance or sensitization [54]. The fact that methylphenidate suppressed social play independently of the baseline levels of play [54] suggests that the effects of this drug on social play cannot be explained by a rate-dependency hypothesis. A more probable explanation is that methylphenidate reduced social play through a mechanism responsible for its therapeutic efficacy in ADHD, such as by improving behavioral inhibition [61], thus suppressing energetic behaviors that are associated with reduced attention to the environment. The effects of methylphenidate on social play were mediated by a noradrenergic mechanism: they were mimicked by the selective norepinephrine uptake inhibitor atomoxetine, which is also used for the treatment of $\mathrm{ADHD}$, but not by either a dopamine reuptake inhibitor or a dopamine receptor agonist [54]. Furthermore, the play-suppressant effects of methylphenidate and atomoxetine were blocked by the $\alpha-2$ adrenoceptor antagonist RX821002, but the effects of methylphenidate were not antagonized by blockade of $\alpha-1$ adrenergic, $\beta$-adrenergic or dopamine receptors [54].

\section{Concluding remarks}

Social play behavior is a specific category of social interaction that is most abundant between weaning and puberty [1-3]. It is of great importance for the development of a flexible behavioral repertoire, in both social and non-social domains, and for the maintenance of group cohesion [3,7]. Social play is very rewarding [11-22]. A number of neurotransmitter systems have been implicated in reward processes, with some specificity in the subcomponents of reward they modulate (hedonics, motivation, cognition). Dopaminergic neurotransmission is important for the motivational properties of rewards, whereas opioids and cannabinoids, depending on their neural site of action, mediate both hedonics and motivation [36-40]. Cognitive properties of rewards are modulated, on different neural levels, by opioids, cannabinoids, dopamine and norepinephrine. These neurotransmitter systems have also been implicated in social play behavior.

Stimulation of $\mu$-opioid receptors enhances social play, most probably by increasing the hedonic properties of social play [17,28-30,35]. Enhancing endocannabinoid neurotransmission also stimulates social play, and blockade of CB1 cannabinoid receptors attenuates the play-stimulating effects of morphine, ethanol and nicotine [32-34,49]. Akin to the neuropharmacological modulation of food and drug reward [47-48], opioid and cannabinoid neurotransmission interact in the modulation of social play behavior [32]. What still needs to be determined is whether these opioid-cannabinoid interactions involve cannabinoid-induced synthesis and release of endogenous opioids (or vice versa), converging intracellular signal transduction pathways through competition for the same pool of Gi-proteins, or direct CB1- $\mu$ receptor heterodimerization at the level of the cell membrane [62]. Dopaminergic neurotransmission modulates the augmenting effects of endocannabinoids, ethanol and nicotine (but remarkably, not opioids) on play $[32,49]$. Last, both noradrenergic [54] and opioid neurotransmission $[30,35]$ have been suggested to modulate certain cognitive aspects of the performance of social play behavior. The effect of methylphenidate on social play, mediated through $\alpha-2$ adrenoceptors, might be the result of improved behavioral inhibition, causing attenuation of vigorous patterns of behavior that are accompanied by reduced attention for the environment. Somewhat related, the role of $\mu$ - and $\kappa$-opioid receptors in reducing the suppression of social play in a novel environment could be the result of a redistribution of attentional resources, shifting behavior away from exploring a novel environment (and its potential threats and opportunities) towards engaging in a pleasurable interaction with an individual (of the same species). Indeed, opioid neurotransmission does play a part in the modulation of attention and working memory [63,64], which might underlie these effects. 
There are two particular lines of research that are essential to fill in the gaps in our knowledge. First, although systemic pharmacological studies can be suggestive of the brain regions and circuits involved, our knowledge of the neuroanatomy of social play behavior is still limited [2] Second, although the hypothesis that opioid, dopaminergic, cannabinoid and noradrenergic neurotransmission, mediate the hedonic, motivational and cognitive properties of social play through dissociable neural circuits is very appealing, this should be tested using behavioral tasks that specifically measure these distinct aspects of social play.

To conclude, our knowledge of the neural mechanisms of social play behavior has markedly increased over the past decades, showing that neurotransmitter systems involved in food and drug reward also modulate the positive subjective properties of social play. Elucidating the neural substrates of social play behavior and its rewarding properties will help us to understand how positive social signals are processed in the brain. Furthermore, comparing the neurobiology of the hedonic, motivational and cognitive aspects of natural (food, sex, social play) and artificial rewards (drugs of abuse) will shed light on how different rewards exert their effects on behavior through common and unique neural pathways. Last, understanding the neurobiology of social play behavior might contribute to the development of novel treatments for psychiatric disorders in which social play is impaired, such as autism, early-onset schizophrenia and ADHD [4-6].

\section{Acknowledgements}

This work was upported by a National Institute on Drug Abuse Grant (R01 DA022628 to L.J.M.J.V.). Part of our research described in this paper was performed within the framework of project T5-107 of the Dutch Top Institute Pharma. We thank Ewoud Schmidt for making the pictures in Figure 1.

\section{References}

1 Panksepp, J. et al. (1984) The psychobiology of play: theoretical and methodological perspectives. Neurosci. Biobehav. Rev. 8, 465-492

2 Vanderschuren, L.J.M.J. et al. (1997) The neurobiology of social play behavior in rats. Neurosci. Biobehav. Rev. 21, 309-326

3 Pellis, S.M. and Pellis, V. (2009) The Playful Brain: Venturing to the Limits of Neuroscience, Oneworld Publications

4 Jordan, R. (2003) Social play and autistic spectrum disorders: a perspective on theory, implications and educational approaches. Autism 7, 347-360

5 Alessandri, S.M. (1992) Attention, play, and social behavior in ADHD preschoolers. J. Abnorm. Child Psychol. 20, 289-302

6 Moller, P. and Husby, R. (2000) The initial prodrome in schizophrenia: searching for naturalistic core dimensions of experience and behavior. Schizophr. Bull. 26, 217-232

7 Špinka, M. et al. (2001) Mammalian play: training for the unexpected. Q. Rev. Biol. 76, 141-168

8 Van den Berg, C.L. et al. (1999) Play is indispensable for an adequate development of coping with social challenges in the rat. Dev. Psychobiol. 34, 129-138

9 Von Frijtag, J.C. et al. (2002) Individual housing during the play period results in changed responses to and consequences of a psychosocial stress situation in rats. Dev. Psychobiol. 41, 58-69

10 Siviy, S.M. (1998) Neurobiological substrates of play behavior: Glimpses into the structure and function of mammalian playfulness. In Animal Play: Evolutionary, Comparative, and Ecological Perspectives (Bekoff, M. and Byers, J.A., eds), pp. 221-242, Cambridge University Press

11 Humphreys, A.P. and Einon, D.F. (1981) Play as a reinforcer for mazelearning in juvenile rats. Anim. Behav. 29, 259-270
12 Calcagnetti, D.J. and Schechter, M.D. (1992) Place conditioning reveals the rewarding aspect of social interaction in juvenile rats. Physiol. Behav. 51, 667-672

13 Thiel, K.J. et al. (2008) Social reward-conditioned place preference: A model revealing an interaction between cocaine and social context rewards in rats. Drug Alcohol Depend. 96, 202-212

14 Thiel, K.J. et al. (2009) Synergistic interaction between nicotine and social rewards in adolescent male rats. Psychopharmacology 204, 391-402

15 Trezza, V. et al. (2009) Conditioned place preference induced by social play behavior: parametrics, extinction, reinstatement and disruption by methylphenidate. Eur. Neuropsychopharmacol. 19, 659-669

16 Ikemoto, S. and Panksepp, J. (1992) The effects of early social isolation on the motivation for social play in juvenile rats. Dev. Psychobiol. 25, 261-274

17 Normansell, L. and Panksepp, J. (1990) Effects of morphine and naloxone on play-rewarded spatial discrimination in juvenile rats. Dev. Psychobiol. 23, 75-83

18 Crowder, W.F. and Hutto, C.W., Jr (1992) Operant place conditioning measures examined using two nondrug reinforcers. Pharmacol. Biochem. Behav. 41, 817-824

19 Van den Berg, C.L. et al. (1999) Isolation changes the incentive value of sucrose and social behaviour in juvenile and adult rats. Behav. Brain. Res. 106, 133-142

20 Douglas, L.A. et al. (2004) Rewarding properties of social interactions in adolescent and adult male and female rats: impact of social versus isolate housing of subjects and partners. Dev. Psychobiol. 45, 153-162

21 Falk, J.L. (1958) The grooming behavior of the chimpanzee as a reinforcer. J. Exp. Anal. Behav. 1, 83-85

22 Mason, W.A. et al. (1963) Preferential responses of young chimpanzees to food and social rewards. Psychol. Rec. 13, 341-345

23 Cheng, S.Y. and Delville, Y. (2009) Vasopressin facilitates play fighting in juvenile golden hamsters. Physiol. Behav. 98, 242-246

24 Guard, H.J. et al. (2002) Morphine administration selectively facilitates social play in common marmosets. Dev. Psychobiol. 41, $37-49$

25 Burgdorf, J. et al. (2008) Ultrasonic vocalizations of rats (Rattus norvegicus) during mating, play, and aggression: Behavioral concomitants, relationship to reward, and self-administration of playback. J. Comp. Psychol. 122, 357-367

26 Panksepp, J. et al. (1980) Endogenous opioids and social behavior. Neurosci. Biobehav. Rev. 4, 473-487

27 Depue, R.A. and Morrone-Strupinsky, J.V. (2005) A neurobehavioral model of affiliative bonding: implications for conceptualizing a human trait of affiliation. Behav. Brain Sci. 28, 313-350; discussion 350-395

28 Panksepp, J. et al. (1985) Opiates and play dominance in juvenile rats. Behav. Neurosci. 99, 441-453

29 Vanderschuren, L.J.M.J. et al. (1995) Sequential analysis of social play behavior in juvenile rats: effects of morphine. Behav. Brain. Res. 72, 89-95

30 Vanderschuren, L.J.M.J. et al. (1995) Effects of morphine on different aspects of social play in juvenile rats. Psychopharmacology 117, 225231

31 Beatty, W.W. and Costello, K.B. (1982) Naloxone and play fighting in juvenile rats. Pharmacol. Biochem. Behav. 17, 905-907

32 Trezza, V. and Vanderschuren, L.J.M.J. (2008) Bidirectional cannabinoid modulation of social behavior in adolescent rats. Psychopharmacology 197, 217-227

33 Trezza, V. and Vanderschuren, L.J.M.J. (2008) Cannabinoid and opioid modulation of social play behavior in adolescent rats: differential behavioral mechanisms. Eur. Neuropsychopharmacol. 18, 519-530

34 Trezza, V. and Vanderschuren, L.J.M.J. (2009) Divergent effects of anandamide transporter inhibitors with different target selectivity on social play behavior in adolescent rats. J. Pharmacol. Exp. Ther. 328, 343-350

35 Vanderschuren, L.J.M.J. et al. (1995) Mu- and kappa-opioid receptormediated opioid effects on social play in juvenile rats. Eur. J. Pharmacol. 276, 257-266

36 Berridge, K.C. and Kringelbach, M.L. (2008) Affective neuroscience of pleasure: reward in humans and animals. Psychopharmacology 199, 457-480

37 Berridge, K.C. et al. (2009) Dissecting components of reward: 'liking', 'wanting', and learning. Curr. Opin. Pharmacol. 9, 65-73 
38 Berridge, K.C. (2007) The debate over dopamine's role in reward: the case for incentive salience. Psychopharmacology 191, 391-431

39 Barbano, M.F. and Cador, M. (2007) Opioids for hedonic experience and dopamine to get ready for it. Psychopharmacology 191, 497-506

40 Kelley, A.E. et al. (2005) Corticostriatal-hypothalamic circuitry and food motivation: integration of energy, action and reward. Physiol. Behav. 86, 773-795

41 Vanderschuren, L.J.M.J. et al. (1995) Social play alters regional brain opioid receptor binding in juvenile rats. Brain Res. 680, 148-156

42 Genn, R.F. et al. (2004) Unconditioned and conditioned anxiogenic effects of the cannabinoid receptor agonist CP 55,940 in the social interaction test. Pharmacol. Biochem. Behav. 77, 567-573

43 van Ree, J.M. et al. (1984) delta 1-Tetrahydrocannabinol but not cannabidiol reduces contact and aggressive behavior of rats tested in dyadic encounters. Psychopharmacology 84, 561-565

44 Pacher, P. et al. (2006) The endocannabinoid system as an emerging target of pharmacotherapy. Pharmacol. Rev. 58, 389-462

45 Egerton, A. et al. (2006) Cannabinoids and prefrontal cortical function: insights from preclinical studies. Neurosci. Biobehav. Rev. 30, 680 695

46 Schneider, M. and Koch, M. (2002) The cannabinoid agonist WIN $55,212-2$ reduces sensorimotor gating and recognition memory in rats. Behav Pharmacol. 13, 29-37

47 Fattore, L. et al. (2004) Cannabinoids and reward: interactions with the opioid system. Crit. Rev. Neurobiol. 16, 147-158

48 Solinas, M. and Goldberg, S.R. (2005) Motivational effects of cannabinoids and opioids on food reinforcement depend on simultaneous activation of cannabinoid and opioid systems. Neuropsychopharmacology 30, 2035-2045

49 Trezza, V. et al. (2009) Prosocial effects of nicotine and ethanol in adolescent rats through partially dissociable neurobehavioral mechanisms. Neuropsychopharmacology 34, 2560-2573

50 Varlinskaya, E.I. and Spear, L.P. (2009) Ethanol-induced social facilitation in adolescent rats: role of endogenous activity at mu opioid receptors. Alcohol Clin. Exp. Res. 33, 991-1000

51 Beatty, W.W. et al. (1984) Suppression of play fighting by amphetamine: effects of catecholamine antagonists, agonists and synthesis inhibitors. Pharmacol. Biochem. Behav. 20, 747-755
52 Niesink, R.J. and Van Ree, J.M. (1989) Involvement of opioid and dopaminergic systems in isolation-induced pinning and social grooming of young rats. Neuropharmacology 28, 411-418

53 Siviy, S.M. et al. (1996) D2 dopamine receptor involvement in the rough-and-tumble play behavior of juvenile rats. Behav. Neurosci. 110, 1168-1176

54 Vanderschuren, L.J.M.J. et al. (2008) Methylphenidate disrupts social play behavior in adolescent rats. Neuropsychopharmacology 33, 29462956

55 Beatty, W.W. et al. (1982) Psychomotor stimulants, social deprivation and play in juvenile rats. Pharmacol. Biochem. Behav. 16, 417-422

56 Thor, D.H. and Holloway, W.R., Jr (1983) Play soliciting in juvenile male rats: effects of caffeine, amphetamine and methylphenidate. Pharmacol. Biochem. Behav. 19, 725-727

57 Homberg, J.R. et al. (2007) Acute and constitutive increases in central serotonin levels reduce social play behaviour in peri-adolescent rats. Psychopharmacology 195, 175-182

58 Panksepp, J., (1993) Rough and tumble play: a fundamental brain process. In Parent-Child Play, pp. 147-184, SUNY Press

59 Normansell, L. and Panksepp, J. (1985) Effects of clonidine and yohimbine on the social play of juvenile rats. Pharmacol. Biochem. Behav. 22, 881-883

60 Siviy, S.M. et al. (1994) Effects of alpha-2 adrenoceptor antagonists on rough-and-tumble play in juvenile rats: evidence for a site of action independent of non-adrenoceptor imidazoline binding sites. Psychopharmacology 113, 493-499

61 Aron, A.R. et al. (2003) Methylphenidate improves response inhibition in adults with attention-deficit/hyperactivity disorder. Biol. Psychiatry $54,1465-1468$

62 Vigano, D. et al. (2005) Molecular and cellular basis of cannabinoid and opioid interactions. Pharmacol. Biochem. Behav. 81, 360-368

63 Itoh, J. et al. (1994) Dynorphin A- potently improves the impairment of spontaneous alternation performance induced by the mu-selective opioid receptor agonist DAMGO in mice. J. Pharmacol. Exp. Ther. $269,15-21$

64 Iordanova, M.D. et al. (2006) Opioid receptors in the nucleus accumbens regulate attentional learning in the blocking paradigm. J. Neurosci. 26, 4036-4045 